\title{
Uso de las herramientas tecnológicas para la enseñanza de la lengua extranjera ${ }^{1}$
}

\section{Use of technological tools for the teaching of the foreign language}

DOI: http://dx.doi.org/10.17981/cultedusoc.9.3.2018.87

Artículo de investigación. Fecha de recepción: 15/06/2018. Fecha de aceptación: 27/11/2018

\author{
Margy Díaz"; \\ Javier Guiacometto y Andrés Cruz ${ }^{3}$ \\ IED Arcesio Cáliz Amador, sede principal (Colombia) \\ madse@hotmail.com
}

Para citar este artículo:

Díaz, M., Guiacometto, J. y Cruz, A. (2018). Uso de las herramientas tecnológicas para la enseñanza de la lengua extranjera. Cultura. Educación y Sociedad 9(3), 741-748. DOI: http://dx.doi.org/10.17981/cultedusoc.9.3.2018.87

\section{Resumen}

El presente proyecto de investigación permitió evidenciar cómo a través de las herramientas tecnológicas se fortalecen el proceso de enseñanza de la lengua extranjera en los estudiantes de Básica primaria. Para el desarrollo del estudio se tuvo en cuenta la guía Kathryn Cronquist y Ariel Fiszbein que examina las mejores prácticas y las áreas de debilidad en América Latina. La investigación es abordada desde el paradigma positivista, de tipo cuantitativo, y con un alcance descriptivo de diseño no experimental. La población son los estudiantes de básica primaria de la Institución educativa Arcesio Cáliz Amador sede principal. Como conclusión se puede inferir que la población encuestada conoce de las herramientas tecnológicas computadores, cel., tabletas entre otros). Y que además logran darle manejo con frecuencia, además conocen de ciertas aplicaciones digitales para que busquen generar nuevos aprendizajes como como es el caso de información de la segunda lengua.

Palabras clave: Herramientas tecnológicas, Lengua extranjera, enseñanza, aprendizaje, aplicaciones digitales.

\section{Abstract}

Present research project made it possible to demonstrate how, through technological tools, the process of teaching the foreign language in primary school students is strengthened. For the development of the study, the guide Kathryn Cronquist and Ariel Fiszbein, who examines the best practices and areas of weakness in Latin America, were taken into account. The research is approached from the positivist paradigm, of a quantitative type, and with a descriptive scope of non-experimental design. The population is the elementary school students of the Arcesio Cáliz Amador educational institution headquarters. As a conclusion it can be inferred that the surveyed population knows of the technological tools computers, cell phones, tablets among others). And also manage to give it frequent, also know of certain digital applications that seek to generate new learning as is the case of information of the second language.

Keywords: Technological tools, foreign language, teaching, learning, digital applications.

\footnotetext{
1 Este artículo ha sido derivado del Programa de Fortalecimiento de la Cultura Ciudadana y Democrática CT+I a través de la IEP apoyada en TIC en el Departamento de Magdalena: CICLON.
}

2 Líder de investigación del grupo "Cool English".

3 Docentes pertenecientes al grupo de investigación “Cool English” de la IED Arcesio Cáliz Amador, sede principal. Ciclón Magdalena, estrategia $\mathrm{N}^{\circ} 2$. Universidad De la Costa CUC.

- The author; licensee Universidad de la Costa - CUC. 


\section{Introducción}

Analizando los diferentes estadios de la vida cotidiana, en notable la necesidad que tenemos actualmente de aprender inglés, esto se pueda dar por diversos motivos, gustos musicales, entretenimiento, por motivos educativos o laborales. En fin hay demasiadas razones por las cuales podemos decir que es necesario aprender este idioma.

Otra manera de argumentar o afirmar la importancia del idioma inglés, es tener presente que es el idioma más hablado y nos permite comunicarnos con otras personas alrededor del mundo. Contar con esta herramienta es de vital importancia debido a que los tiempos han cambiado y hablar este idioma nos puede brindar maravillosas oportunidades laborales, educativas o de otro carácter, pero lo más impactante es que nos puede brindar una buena calidad de vida

El aprendizaje de una segunda lengua, en especial la del inglés, se ha convertido en uno de los más importantes temas hoy en día como estrategia para todos los países de América Latina. Los países latinos han desarrollado estrategias nacionales, creando programas y realizado costosas e innovadoras inversiones para expandir el acceso a las oportunidades de aprender inglés.

En el 2017 se realizó un informe elaborado por Kathryn Cronquist y Ariel Fiszbein para brindar un panorama más amplio del estado del aprendizaje del inglés en diez países de América Latina, enfocándose en evaluar las políticas en curso y en identificar las dificultades clave que afectan las oportunidades para tener programas de alta calidad.

Este informe pretende brindar un panorama del estado del AIE en diez países latinoamericanos: Argentina, Brasil, Chile, Colombia, Costa Rica, Ecuador, México, Panamá, Perú y Uruguay y es una contribución sobre cómo mejorar la calidad del aprendizaje del idioma inglés en los países de habla hispana o latinoamericanos. Se muestran los resultados de distintas pruebas y exámenes indican que el dominio del inglés en estos países es muy bajo.

El sistema educativo simplemente no está generando estudiantes con los niveles necesarios de dominio del inglés. Las escuelas con frecuencia son incapaces de impartir las clases de inglés necesarias, y aquellas que lo hacen suelen impartir clases de baja calidad. Las oportunidades de aprendizaje fuera del sistema educativo no logran compensar las deficiencias.

Por lo tanto el presente proyecto de investigación busca identificar la importancia de herramientas pedagógicas virtuales para la enseñanza de la lengua extranjera-ingles, en los estudiantes de básica primaria.

El informe de Kathryn Cronquist y Ariel Fiszbein examina las mejores prácticas y las áreas de debilidad en América Latina, por medio de un análisis de las políticas de aprendizaje del idioma ingles (AIE), la eficacia de los profesores y los programas de enseñanza del inglés. Por lo tanto, mejorar el aprendizaje del idioma inglés en América Latina requerirá de un marco de políticas que guíe las acciones y las inversiones en el sistema educativo y de políticas para el aprendizaje del idioma ingles en diez países latinoamericanos: Argentina, Brasil, Chile, Colombia, Costa Rica, Ecuador, México, Panamá, Perú y Uruguay.

Se consideran cuatro componentes que, en conjunto, dan forma a los esfuerzos por ofrecer un aprendizaje del inglés dentro del sistema escolar: el fundamento legal, los estándares de aprendizaje del inglés y los apoyos alineados a esos estándares, la medición del desempeño de los estudiantes, y la medición de las cualificaciones de los docentes. Por otro lado, se considera que las políticas son herramientas para la administración y la dirección estratégica, brindan una valiosa perspectiva para entender las fortalezas y debilidades en el nivel sistémico. 
Importancia de la segunda lengua (inglés) en la educación

En américa latina el aprendizaje del inglés se ha convertido en un tema estratégico de alto impacto. Algunos países han llevado a cabo planes estratégicos con relación a este tema, se han realizado grandes inversiones para facilitar las oportunidades que permitan aprender inglés.

Una de las justificaciones más validas de la importancia de hablar varios idiomas es el impacto que tiene el capital humano, puesto que dominar un segundo idioma puede ayudar al crecimiento económico de un país.

En la actualidad el dominar una lengua extranjera y más específicamente el inglés, es una necesidad debido a todos los cambios que han presentado los diferentes estadios los cuales pueden ser: laborales, educativos, culturales, etc. "Este fenómeno ha sido el principal motivo por el cual el inglés ha adquirido importancia a nivel mundial, hasta el punto de considerarse hoy en día como el idioma universal de los negocios" (Uribe, 2012).

En última década se le ha dado prioridad al aprendizaje del idioma ingles y es por ello que entidades como el Ministerio de Educación Nacional, se han visto en la tarea de crear el programa de Bilingüismo, como estrategia para la competitividad 2004-2019, el cual tiene como objetivo alcanzar la meta de que los estudiantes de las instituciones de educación básica, media, superior y de estudios no formales adquieran competencias del habla inglés.

El Gobierno Nacional ha tomado iniciativas dedicadas a fomentar el aprendizaje del inglés y a mejorar la calidad en la enseñanza del mismo, siendo el Programa Nacional de Bilingüismo (PNB) la principal de estas iniciativas. El PNB fue establecido en 2004 por el MEN, no solo como un instrumento que busca contribuir a mejorar la calidad educativa, sino como una estrategia para la promoción de la competitividad.
Según los autores, la implementación del PNB se basa en la importancia que tiene el dominio de una lengua extranjera, con la cual la sociedad puede participar de manera activa en las actividades que están relacionadas al crecimiento académico, tecnológico y cultural. "El mejoramiento de la competencia comunicativa en inglés conlleva al surgimiento de oportunidades para sus ciudadanos, el reconocimiento de otras culturas y el crecimiento individual y colectivo" (Sanchez, 2013).

El dominio del inglés es cada vez más necesario para los negocios y la comunicación internacional; en ese sentido, se vincula con prospectos de competitividad económica y crecimiento en la economía global. El interés por aprender el idioma continúa en ascenso en toda América Latina. La región ha hecho considerables esfuerzos para mejorar el aprendizaje del inglés por medio de políticas y programas, lo que ha dado como resultado que más personas en la región tienen acceso al Aprendizaje del Idioma Inglés (AIE).

Respecto a los estudiantes de educación media y estudios no formales, el objetivo es que al culminar sus estudios posean un manejo básico del inglés tanto hablado como escrito. Pues, en Colombia el propósito de dominio de la segunda lengua está encaminado a lograr que los ciudadanos posean la capacidad de comunicarse en inglés, "de tal forma que se puedan insertar al país en los procesos de comunicación universal, en la economía global y en la apertura cultural, con estándares internacionales comparables" (Uribe, 2012).

El aprendizaje del inglés, como lengua extranjera, ha resultado complejo, aunque se reconozca que su adquisición ofrece la ventaja de facilitarle muchas salidas profesionales Posiblemente, como resultado de lo anterior, las clases más demandadas por los estudiantes tanto jóvenes como adultos son las de informática e inglés. 
En general, en el aprendizaje de un idioma extranjero los obstáculos fundamentales que encuentran las personas se derivan del estilo de metodología utilizada por el docente (Ricoy y Alvarez, 2016).

"A pesar de existir una gran diversidad no es frecuente la utilización de metodologías innovadoras o que le resulten atractivas al alumnado" (Ricoy y Alvarez, 2016).

\section{Estrategias para el aprendizaje del inglés}

Es de vital importancia tener en cuenta que para lograr el objetivo de aprender una lengua extranjeras se necesita que la persona practique continuamente e interactúe con otras personas, y que se encuentren en contextos reales, lo que les facilitará el proceso de aprendizaje.

El aprendizaje del inglés requiere de contextos que le brinde al estudiante situaciones cotidianas que le faciliten la consolidación de los conocimientos. La adquisición de una lengua extranjera trae consigo unos elementos indispensables a tener en cuenta en donde los estudiantes deben realizar procesos de apropiación y dominio consciente de la misma.

Cabe resaltar que para el aprendizaje de idiomas se debe fortalecer el desarrollo de la competencias orales, escritas y auditivas, dándole privilegio a las estrategias que respondan a los desafíos y dificultades en relación al seguimiento, proceso y evaluación de lo que se imparte y lo que se aprende.

"Existen factores internos en los estudiantes que influyen en el proceso de aprendizaje de una segunda lengua; es posible que estos factores estén relacionados con las estructuras ya adquiridas de la lengua materna, o también puede que estén relacionados con la habilidad propia de cada persona para aprender un idioma" (Moya y Jiménez 2004)
Uno de los métodos más relevantes para la enseñanza de un segundo idioma son los apoyados en estrategias didácticas alternativas y en las TICs. En este caso, pueden referirse aquellos que abogan por el aprendizaje globalizado y el aprendizaje del lenguaje en comunidad.

"En ellos la inclusión de los estímulos externos que incorporen componentes lúdicos y vinculados con la cotidianidad así como la combinación de recursos didácticos novedosos que tienen como finalidad mejorar el interés por el idioma a adquirir" (Ricoy y Alvarez, 2016).

Actualmente, los métodos y recursos didácticos empleados para el aprender una lengua extranjera en las Instituciones Educativas todavía no han producido los logros deseados. De hecho, las carencias y lagunas que presenta gran parte de la población son manifiestas, también es evidente la resistencia al cambio que se genera con relación al uso de nuevas tecnologías para el aprendizaje, esto se puede dar por la complejidad y limitaciones que presenta dicho proceso.

En concordancia con lo expuesto por Aguilar (2015), en donde manifiesta que el trabajo grupal y colaborativo, promueve a la aplicación de estrategias particulares, mientras que otros factores pueden traer consigo resultados negativos de acuerdo a lo que se vivencia en el colectivo.

"A la hora de aprender un idioma, la actitud o disposición que la persona presente es clave y, del mismo modo, la motivación intrínseca y extrínseca resulta sustancial" (Ordoñez, 2001).

Por ello, el proceso de aprendizaje de los jóvenes y adultos debe ser diferente, el instructor debe implementar estrategias de motivación para el grupo desde el inicio, y crear un clima adecuado para gestionar el deseo de aprender, además aclarando su utilidad cotidiana, así mismo en los ámbitos académicos y productivos. La generación de un ambiente agradable en 
el aula, que posibilite la desinhibición a las personas jóvenes y adultas para evitar que se retraigan o avergüencen, al hablar o para preguntar promoverá una mejor comunicación.

El docente es el factor clave en este punto, el cual puede propiciar la generación de actividades interactivas, a partir de la integración de diversos recursos didácticos y si logra hacerles conscientes del progreso en el aprendizaje de la lengua extranjera.

"Otra característica esencial para que la motivación llegue a consolidarse de manera exitosa es a través de la combinación de prácticas y recursos muy variados y novedosos que contribuyan a que el alumnado no pierda la atención" (Rubio 2011)

"Así como estableciendo vínculos congruentes entre la formación académica y su experiencia vital" (Goethals, 2014).

"De modo que deberán evitarse las actividades repetitivas y mecánicas, ya que conducen a la monotonía o aburrimiento y pueden causar apatía o desmotivación" (Cortés, y Cabrera.2003).

\section{Rol del maestro en el aprendizaje de una segunda lengua}

Es esencial contar con profesores de calidad para mejorar los niveles de dominio del inglés, puesto que ellos son los responsables de implementar cada día las políticas y actividades. Sin embargo, la enseñanza del inglés ha mostrado debilidades relacionadas tanto con el dominio del inglés por parte de los profesores como con la calidad de su capacitación (Pérez \& Sola, 2004).

En america latina actualmente existe una alta demanda de profesores instructores; por otro lado las oportunidades de capacitaciones para ellos son abundantes, pero esto no garantiza la calidad de las capacitaciones y por lo general son deficientes. Existen sistemas de acreditación para garantizar la calidad, pero hay muchos programas que no son acreditados y estos continúan con sus actividades, inscribiendo alumnos e impartiendo clases.

También hay una amplia variabilidad en el uso de requisitos de ingreso o egreso, y pocos países tienen un enfoque estandarizado. Además, las opciones de desarrollo profesional para los maestros de inglés enfrentan problemas de evaluación y continuidad. En toda la región existen oportunidades de capacitarse tanto dentro de cada país como en el extranjero (Cronquist y Fiszbein. 2017; Camacho., Ordóñez, Fernández., García y Dorado 2014).

\section{Las TIC y su importancia en el aprendizaje de una segunda lengua}

Castellanos (2015), plantea que es un tema relevante en la actualidad. Es un catalizador de cambio, al mejorar el desempeño estudiantil y promover el trabajo colaborativo. Las Tics no solamente promueven un mejor rendimiento escolar sino el aprendizaje colaborativo entre los educandos y los miembros de la familia, así mismo el empoderamiento en el fortalecimiento del cuidado del medio ambiente y la toma de conciencia para mejorar las condiciones de vida de la comunidad.

Una de las integraciones de las TIC en los temas de la educación mayormente puesta en práctica en la actualidad se da en el área de idiomas con el nombre de aprendizaje de lenguas asistido por computadora, como estrategia novedosa que se origina a partir de la angustia de los científicos por integrar las TIC en todos los procesos de enseñanza aprendizaje de los idiomas distintos al materno.

"Por tanto, las tecnologías que se utilizan con mayor frecuencia en los niveles educativos son la computadora, el correo electrónico y la Internet, y su aplicación puede ser tan variada como permitan las circunstancias de cada plantel” (Arteaga, 2011). 
Otra de las innovaciones de las TIC ha sido colocadas a prueba por los mismos estudiantes, quienes hacen uso de los medios tecnológicos disponibles y cada vez más sofisticados, por necesidad o moda, y son ellos quienes han buscado incursionar en la aplicación de estas herramientas.

\section{Metodología}

El presente proyecto de investigación, se realizó tomando las bases de paradigma positivista, desde una mirada cuantitativa, con un alcance descriptivo. Desde un diseño no experimental.

\section{Población y muestra}

Para la realización del estudio se utilizó una población de (30) estudiantes de básica primaria de la Institución Educativa Departamental Arcesio Cáliz Amador sede principal.

\section{Técnicas e instrumentos}

de recolección de información

Se realizó una encuesta de 9 preguntas que buscan identificar las capacidades de los estudiantes en el uso de herramientas tecnológicas para el aprendizaje de inglés.

\section{Procedimiento}

Fase (1): Identificación de la problemática a estudiar.

Fase (2): Definición de la población y muestra.

Fase (3): Definición de las variables de estudio.

Fase (4): Fundamentación teórica.

Fase (5): Escogencia de las técnicas e instrumentos de recolección de información.

Fase (6): Aplicación de los instrumentos.

Fase (7): Análisis y procesamiento de los datos.

Fase (8): Generación de resultados.

Fase (9): Socialización y discusión de los hallazgos encontrados.

\section{Resultados}

A continuación, se exponen los resultados derivados de la aplicación de la encuesta que consta de 9 preguntas relacionadas directamente con las capacidades en el uso de herramientas tecnológicas para el aprendizaje de inglés.

\section{Conclusión}

A modo de conclusión se puede inferir que la población encuestada conoce de las herramientas tecnológicas (computadores, cel., tabletas entre otros). Y que además logran darle manejo con frecuencia, resaltando que entran a navegar en internet, manejan los canales de comunicación en red (WhatsApp, Messenger, correo).

Por otro lado, conocen de ciertas aplicaciones digitales para que busquen generar nuevos aprendizajes como como es el caso de información de la segunda lengua.

En la muestra representativa, el resultado tras la aplicación de la prueba positiva, en su mayoría arrojo un puntaje alto, en conocimiento sobre varias pruebas, que de acuerdo al grado que cursan es de su interés.

\section{Referencias}

Aguilar, J. (2015). Programas educativos compensatorios en México. Problemas de equidad y de conocimiento, Perfiles Educativos (México), 37(147). 183-200.

Arteaga, C. (2011). Uso de las TIC para el aprendizaje del inglés en la Universidad Autónoma de Aguascalientes. Apertura, $3(2)$.

Betancur, S., Carmona, L., Contreras, R., Karam, J., Maestre, N., Romero, Y. y Uribe, S. (2014). Videojuegos y tic como Estrategias Pedagógicas: Formación para el uso seguro de internet. Cultura Educación y Sociedad, 5(1). 91-107. Recuperado de https:/revistascientificas.cuc.edu.co/ culturaeducacionysociedad/article/ view/997 


\begin{tabular}{|c|c|c|c|}
\hline $\mathrm{N}^{\circ}$ & Ítems & $\begin{array}{c}\text { Resultado } \\
\text { cuantitativo }\end{array}$ & Resultado cualitativo \\
\hline 1 & $\begin{array}{l}\text { ¿Cuál de estos } \\
\text { dispositivos } \\
\text { tecnológicos } \\
\text { (Celular, computador } \\
\text { portátil, tabletas } \\
\text { otros) utilizas con } \\
\text { más frecuencia? }\end{array}$ & $\begin{array}{l}80 \% \\
10 \% \\
0 \%\end{array}$ & $\begin{array}{l}\text { La encuesta mostró que el dispositivo tecnológico } \\
\text { más utilizado por los estudiantes es el celular } \\
\text { con un seguido de computadoras portátiles y } \\
\text { tabletas. Finalmente, se observa una utilización } \\
\text { nula de computadores de mesa. }\end{array}$ \\
\hline 2 & $\begin{array}{l}\text { ¿Con qué frecuencia } \\
\text { consideras } \\
\text { que utilizas } \\
\text { el dispositivo } \\
\text { mencionado } \\
\text { anteriormente? }\end{array}$ & $100 \%$ & $\begin{array}{l}\text { La totalidad de los estudiantes encuestados } \\
\text { manifestaron utilizar el dispositivo electrónico } \\
\text { todos los días, lo cual refleja como el uso de } \\
\text { las tecnologías hace parte de integral de las } \\
\text { actividades diarias. }\end{array}$ \\
\hline 3 & $\begin{array}{l}\text { ¿En cuales lugares } \\
\text { te conectas con } \\
\text { mayor frecuencia a } \\
\text { internet? }\end{array}$ & $\begin{array}{l}70 \% \\
20 \% \\
10 \%\end{array}$ & $\begin{array}{l}\text { El lugar en el cual los estudiantes acceden con } \\
\text { mayor frecuencia a internet es sus casas, seguido } \\
\text { del colegio y finalmente el café internet. }\end{array}$ \\
\hline 4 & $\begin{array}{l}\text { ¿Cuál es la } \\
\text { frecuencia con la que } \\
\text { usted participa en } \\
\text { las redes sociales? }\end{array}$ & $\begin{array}{l}90 \% \\
10 \%\end{array}$ & $\begin{array}{l}\text { Los estudiantes en su mayoría participan en las } \\
\text { redes sociales, y solo una minoría dice que lo } \\
\text { hace algunas veces. }\end{array}$ \\
\hline 5 & $\begin{array}{l}\text { ¿Qué nivel de } \\
\text { participación tienes } \\
\text { en estas redes } \\
\text { sociales: Facebook/ } \\
\text { Instagram/ } \\
\text { WhatsApp? }\end{array}$ & $\begin{array}{l}90 \% \\
10 \%\end{array}$ & $\begin{array}{l}\text { S6e encontró que la mayoría de los participantes } \\
\text { tiene una participación frecuente en estos medios } \\
\text { soci8ales y solo un porcentaje bajo afirmo que } \\
\text { utiliz9a estas redes sociales algunas veces. }\end{array}$ \\
\hline 6 & $\begin{array}{l}\text { ¿Qué grado de } \\
\text { conocimiento tienes } \\
\text { sobre la siguiente } \\
\text { herramienta } \\
\text { tecnológica? } \\
\text { "Skype". }\end{array}$ & $\begin{array}{l}60 \% \\
30 \% \\
10 \%\end{array}$ & $\begin{array}{l}\text { Más de la mitad de la población entrevistada } \\
\text { tiene desconocimiento de la herramienta } \\
\text { "Skype", seguido de un grupo que tiene } \\
\text { conocimientos medios y solo un grupo pequeño } \\
\text { conoce a profundidad este medio. Nulo. }\end{array}$ \\
\hline 7 & $\begin{array}{l}\text { ¿Qué grado de } \\
\text { conocimiento tienes } \\
\text { sobre la herramienta } \\
\text { "Power point"? }\end{array}$ & $\begin{array}{l}50 \% \\
40 \% \\
10 \%\end{array}$ & $\begin{array}{l}\text { La mitad de los participantes manifiestan si } \\
\text { conocer la herramienta, en segundo lugar se } \\
\text { encuentra un grupo de estudiantes que poseen } \\
\text { conocimientos medios y finalmente un grupo que } \\
\text { no tiene conocimiento de la herramienta "Power } \\
\text { point". }\end{array}$ \\
\hline 8 & $\begin{array}{l}\text { ¿Qué grado de } \\
\text { conocimiento tienes } \\
\text { sobre la herramienta } \\
\text { tecnológica "Correo } \\
\text { electrónico"? }\end{array}$ & $\begin{array}{l}50 \% \\
40 \% \\
10 \%\end{array}$ & $\begin{array}{l}\text { La mitad de los participantes manifiestan si } \\
\text { conocer la herramienta, en segundo lugar se } \\
\text { encuentra un grupo de estudiantes que poseen } \\
\text { conocimientos medios y finalmente un grupo que } \\
\text { no tiene conocimiento de la herramienta "Correo } \\
\text { electrónico". }\end{array}$ \\
\hline 9 & $\begin{array}{l}\text { ¿Has utilizado } \\
\text { alguna aplicación } \\
\text { para aprender } \\
\text { inglés? }\end{array}$ & $\begin{array}{l}80 \% \\
20 \%\end{array}$ & $\begin{array}{l}\text { La mayoría de los participantes manifiestan } \\
\text { haber utilizado alguna aplicación para aprender } \\
\text { inglés y el resto de estudiantes afirma no haberlo } \\
\text { hecho. }\end{array}$ \\
\hline
\end{tabular}

Fuente: elaboración propia. 
Camacho, E., Ordóñez, G., Fernández, M., García, S. y Dorado, V. (2014). Representaciones sociales, frente al aprendizaje del inglés. [Tesis maestría]. Universidad de Manizales, Manizales. Recuperado de http://ridum.umanizales.edu.co:8080/ xmlui/handle/6789/1862

Cortés, A. y Cabrera, L. (2003). Programa de Inglés para la Conversación Área Comercial y Servicios Secretariado X, XI y XII. Costa Rica: MEP.

Cronquist, K. y Fiszbein, A. (2017). El aprendizaje del inglés en América Latina. Recuperado de https://www.thedialogue.org/ wp-content/uploads/2017/09/El-aprendiz a j e - d e l - i n g l \% C $3 \%$ A 9 s - e n Am\%C3\%A9rica-Latina-1.pdf

España, C. (2010). El idioma inglés en el currículo universitario: importancia, retos y alcances. Educare, 14(2). 63-69.

Goethals, P. (2014). La acomodación lingüística en contextos profesionales turísticos. Un enfoque didáctico basado en los testimonios de turistas. Ibérica (España), (28). 181-202.

Herrera, T. y Suarez, E. (2014). El ambiente de aprendizaje del inglés como lengua extranjera y el comportamiento de los estudiantes entre los nueve y doce años de edad de la Fundación ASE. [Tesis]. Universidad de la Salle, Bogotá D.C., Colombia.

Jiménez, M. y Moya, J. (2004). El proceso de interlengua en el aprendizaje del inglés como lengua extranjera en edades tempranas. Glosas didácticas, revista electrónica ambiente de aprendizaje y comportamiento 117 internacional. (11). 128-142. Disponible en http://www.um.es/ glosasdidacticas/doc-es/10moya.pdf

Marín, F., Niebles, M., Sarmiento, M. y Valbuena, S. (2017). Mediación de las tecnologías de la información en la comprensión lectora para la resolución de problemas aritméticos de enunciado verbal. Espacios, 38(20). Recuperado de http://www.revist a e s pacios.com/a 17 v 38 n $20 /$ a17v38n20p20.pdf
Martìnez, J., Berthel, Y. y Vergara, M. (2017). Síndrome de Burnout en profesores y su relación con el aprendizaje de los estudiantes de básica primaria de una institución educativa oficial de Sincelejo (Colombia), 2016. Salud Uninorte, 33(2). 118-128. http://dx.doi.org/10.14482/sun.33.2.10538

Ordóñez, J. (2001). La globalización de la tecnología, humanismo y educación. Globalización, identidades y educación. Cuadernos Pedagógicos 4. 1-69.

Patiño, A. (2015). Tendencias tecnológicas que influyen en el aumento de la productividad empresarial. INGE CUC, 11(2), 8496. https://doi.org/10.17981/ingecuc.11.2. 2015.09

Pérez, A. y Sola, M. (2004). Investigación e Innovación en la formación del Profesorado. Málaga: Universidad de Málaga.

Ricoy, M. y Álvarez, S. (2016.) La enseñanza del inglés en la educación básica de personas jóvenes y adultas. Revista Mexicana de Investigación Educativa. 21(69). 385- 409

Rubio, M. (2011). Factores que intervienen en el aprendizaje de una segunda lengua. $R e$ vista Digital Innovación y Experiencias Educativas (España), (46). 1-9.

Uribe, J. (2012). Importancia del idioma inglés en las instituciones de educación superior: el caso de la Corporación Universitaria de Sabaneta. Uni-pluri/versidad, 12(2).

Sanchez, A. (2013). Bilingüismo en Colombia. Documento de trabajo sobre economía regional. Documentos de Trabajo sobre Economía Regional, 91. Recuperado de http://www.banrep.gov.co/docum/Lectura_finanzas/pdf/dtser_191.pdf

Castellanos, A. (2015). Aplicación de las tic como herramienta didáctica para la enseñanza - aprendizaje de la técnica básica del balonmano con los niños del grado quinto del colegio nydia quintero de turbay I.E.D. [Tesis].Universidad Libre, Barranquilla. de http://repository.unilibre.edu.co/bitstream/handle/10901/8310/proyecto\%20 de $\% 20$ grado.pdf?sequence $=1$ 